\title{
Reconstruction of Betti numbers of manifolds for anisotropic Maxwell and Dirac systems
}

\author{
Katsiaryna KrupchyK, Yaroslav Kurylev and Matti Lassas
}

\begin{abstract}
We consider an invariant formulation of the system of Maxwell's equations for a general anisotropic medium on a compact orientable Riemannian three-manifold $(M, g)$ with nonempty boundary. The system can be completed to a Dirac type first-order system on the manifold. We show that the Betti numbers of the manifold can be recovered from the dynamical response operator for the Dirac system given on a part of the boundary. In the case of the original physical Maxwell system, assuming that the entire boundary is known, all Betti numbers of the manifold can also be determined from the dynamical response operator given on a part of the boundary. Physically, this operator maps the tangential component of the electric field into the tangential component of the magnetic field on the boundary.
\end{abstract}

\section{Introduction}

Recently there has been a lot of interest in inverse problems for Maxwell's equations in Euclidean domains in $\mathbb{R}^{3}$ and on compact Riemannian manifolds, see $[4,14-16,22-24]$. In a smooth bounded domain $M \subset \mathbb{R}^{3}$, Maxwell's equations are given by

$$
\begin{aligned}
& \operatorname{curl} E(x, t)=-B_{t}(x, t), \\
& \operatorname{curl} H(x, t)=D_{t}(x, t),
\end{aligned}
$$

where $E$ and $H$ are the electric and magnetic fields, and $B$ and $D$ are the magnetic flux density and the electric displacement. The fields $E$ and $D$, and similarly, the fields $H$ and $B$ are related by the constitutive relations,

$$
D(x, t)=\epsilon(x) E(x, t), \quad B(x, t)=\mu(x) H(x, t),
$$

where the electric permittivity $\epsilon(x)$ and the magnetic permeability $\mu(x)$ are $C^{\infty}$-smooth positive-definite $3 \times 3$-matrix valued functions on $M$. The 
initial boundary value problem for the time dependent Maxwell's equations consists of (1.1) and (1.2) together with the conditions

$$
\begin{array}{r}
\left.E(x, t)\right|_{t=-\tau_{f}}=0,\left.\quad H(x, t)\right|_{t=-\tau_{f}}=0, \\
n \times\left. E\right|_{\partial M \times \mathbb{R}_{-}}=f,
\end{array}
$$

where $n$ is the unit exterior normal to $\partial M$, and $\tau_{f}>0$ is such that $f(x, t)=0$ for $t<-\tau_{f}$. The inverse problem associated with (1.1) to (1.3), is the problem of reconstruction of electromagnetic parameters $\epsilon(x)$ and $\mu(x)$ from the knowledge of the response operator

$$
R: n \times\left. E\right|_{\partial M \times \mathbb{R}_{-}} \mapsto n \times\left. H\right|_{\partial M \times \mathbb{R}_{-}} .
$$

From the point of view of modern electrodynamics and classical field theories, it is natural to adopt an invariant approach to Maxwell's equations, where the domain $M$ is replaced by a general three-dimensional smooth compact oriented connected Riemannian manifold, and the vector fields $E$, $H, D$ and $B$ are viewed as differential forms, see [27]. The geometric inverse problem is then to determine the unknown manifold $M$, together with the electromagnetic parameters, from the response operator (1.4), which is now defined in terms of boundary traces of the corresponding differential forms. See also [17-19], where the problem of the reconstruction of a Riemannian manifold from the Dirichlet-to-Neumann operator for harmonic functions, has been studied.

In the context of time-harmonic Maxwell's equations in an isotropic setting, i.e., when the parameters $\epsilon(x)$ and $\mu(x)$ are scalar, the inverse problem for bounded domains in $\mathbb{R}^{3}$ was solved in [22], see also $[6,20,24]$. Much less is known in the anisotropic case. To the best of our knowledge, the positive results in this direction have only been established in the case of an anisotropic medium of a special type, characterized by the polarization independent velocity of the wave propagation. In terms of the electromagnetic parameters, this amounts to the existence of $\alpha(x)>0$ such that $\epsilon(x)=\alpha(x) \mu(x)$. In this case, under a certain geometric condition, it is shown in [14] that, if the conformal class of $\epsilon(x)$ and $\mu(x)$ is known, the stationary boundary measurements identify uniquely the conformal factors. There are also counterexamples for uniqueness of time-harmonic inverse problems involving very anisotropic and degenerate material parameters $[11,12]$. In [16], the inverse problem for Maxwell's equations in the time domain for an anisotropic medium was studied, still assuming that the wave propagation is independent of the polarization. It was shown that the Riemannian manifold and the electromagnetic parameters can be recovered 
from the dynamical response operator similar to (1.4), given on a finite time interval. See also [1] for reconstruction of the wave speed.

In this paper we shall be concerned with the case of a general anisotropic medium. Specifically, working in the geometric setting of Maxwell's equations on a manifold $M$, we are able to recover the Betti numbers of the manifold from the dynamical response operator, given on an open subset of the boundary. This can be viewed as the first step in attempting to reconstruct the geometry and topology of the underlying manifold, in the full generality of the anisotropic case. Let us remark that in the isotropic case, as well as in the case when $\epsilon(x)=\alpha(x) \mu(x), \alpha(x)>0$, the reconstruction of the manifold and the electromagnetic parameters is based on controllability results, which in turn rely crucially on generalizations of the Tataru unique continuation theorem $[9,16]$. In our opinion, the main obstacle in the study of the inverse problem for the general anisotropic Maxwell system is due to the fact that such unique continuation results do not seem to be available in this case.

We would like also to mention the paper [2], where the reconstruction of the Betti numbers of a manifold from the Dirichlet-to-Neumann operator for the Hodge Laplacian on differential forms is studied.

The plan of the paper is as follows. Section 2 is devoted to the description of our geometric setup, including the completion of the Maxwell system to a Dirac type elliptic system, and contains the statement of the main results. We also discuss examples that illustrate the significance of our results for the determination of the topological structure of an unknown object from the boundary measurements. In Section 3, we prove the identifiability of the Betti numbers in the complete Maxwell case, while in Section 4 we establish our results for the physical Maxwell system.

\section{Preliminaries and statement of the main results}

\subsection{Invariant definition of Maxwell's equations}

Let $\left(M, g_{0}\right)$ be a smooth compact oriented connected Riemannian threemanifold with $\partial M \neq \emptyset$. We shall first rewrite equations (1.1) and (1.2), in the anisotropic case, using the language of differential forms. In doing so, we shall follow closely [16], where the case $\epsilon(x)=\alpha(x) \mu(x), \alpha(x)>0$, is considered.

Let $\Lambda^{k} T^{*} M, k=0,1, \ldots, 3$, be the bundle of the $k$ th exterior differential forms and $\Lambda T^{*} M$ be the full bundle of differential forms. Denote by $C^{\infty}\left(M, \Lambda^{k} T^{*} M\right)$ the space of smooth real exterior differential forms of degree $k$. 
Define the fiberwise duality between one-forms and vector fields,

$$
{ }^{\text {b }:} C^{\infty}(M, T M) \rightarrow C^{\infty}\left(M, \Lambda^{1} T^{*} M\right), \quad X^{b}(Y)=g_{0}(X, Y),
$$

or in a coordinate system for $X=a^{i} \frac{\partial}{\partial x^{i}}, X^{b}=g_{0, i j} a^{j} d x^{i}$. This map is bijective and has the following properties [26]:

$$
(\operatorname{curl} X)^{b}=*_{0} d X^{b}, \quad \operatorname{div} X=-*_{0} d *_{0} X^{b}
$$

where

$$
d: C^{\infty}\left(M, \Lambda^{k} T^{*} M\right) \rightarrow C^{\infty}\left(M, \Lambda^{k+1} T^{*} M\right)
$$

is the exterior differential and $*_{0}$ is the Hodge operator with respect to the metric $g_{0}$, acting fiberwise,

$$
*_{0}: C^{\infty}\left(M, \Lambda^{k} T^{*} M\right) \rightarrow C^{\infty}\left(M, \Lambda^{3-k} T^{*} M\right) .
$$

We define the one-forms $\mathcal{E}=E^{b}$ and $\mathcal{H}=H^{b}$ and the two-forms $\mathcal{B}=*_{0} B^{b}$ and $\mathcal{D}=*_{0} D^{b}$. Using the identity $*_{0} *_{0}=\mathrm{id}$, valid in the three-dimensional case, we can write Maxwell's equations (1.1) in terms of differential forms as

$$
d \mathcal{E}=-\partial_{t} \mathcal{B}, \quad d \mathcal{H}=\partial_{t} \mathcal{D}
$$

Consider now the constitutive relations (1.2). We shall determine a metric $g_{\epsilon}$ such that the Hodge operator with respect to this metric, denoted by $*_{\epsilon}$, satisfies

$$
\mathcal{D}=*_{0}(\epsilon E)^{b}=*_{\epsilon} \mathcal{E}
$$

In local coordinates $\left(x^{1}, x^{2}, x^{3}\right)$, we have $\epsilon E=\epsilon_{k}^{i} E^{k} \frac{\partial}{\partial x^{i}},(\epsilon E)^{b}=g_{0, i j} \epsilon_{k}^{j} E^{k} d x^{i}$ and thus, the middle term of (2.2) yields

$$
\begin{aligned}
*_{0}(\epsilon E)^{b} & =*_{0}\left(g_{0, i j} \epsilon_{k}^{j} E^{k} d x^{i}\right)=\frac{1}{2} \sqrt{\operatorname{det}\left(g_{0}\right)} g_{0}^{i l} g_{0, i j} \epsilon_{k}^{j} E^{k} s_{l p q} d x^{p} \wedge d x^{q} \\
& =\frac{1}{2} \sqrt{\operatorname{det}\left(g_{0}\right)} \epsilon_{k}^{j} E^{k} s_{j p q} d x^{p} \wedge d x^{q}
\end{aligned}
$$

where $s_{l p q}$ is the Levi-Civita permutation symbol. Here we may recall that $s_{l p q}=1$ if $(l, p, q)$ is an even permutation of $(1,2,3), s_{l p q}=-1$ if $(l, p, q)$ is an odd permutation, and $s_{l p q}=0$ if at least two indices are the same. 
The right hand side of (2.2) implies that

$$
*_{\epsilon} \mathcal{E}=*_{\epsilon}\left(g_{0, i k} E^{k} d x^{i}\right)=\frac{1}{2} \sqrt{\operatorname{det}\left(g_{\epsilon}\right)} g_{\epsilon}^{i j} g_{0, i k} E^{k} s_{j p q} d x^{p} \wedge d x^{q}
$$

Hence, (2.2) is valid if we set

$$
\sqrt{\operatorname{det}\left(g_{\epsilon}\right)} g_{\epsilon}^{i j} g_{0, i k}=\sqrt{\operatorname{det}\left(g_{0}\right)} \epsilon_{k}^{j}
$$

By taking the determinants of both sides, we obtain

$$
\sqrt{\operatorname{det}\left(g_{\epsilon}\right)}=\operatorname{det}(\epsilon) \sqrt{\operatorname{det}\left(g_{0}\right)}
$$

Defining

$$
g_{\epsilon}^{i j}=\frac{1}{\operatorname{det}(\epsilon)} \epsilon_{k}^{j} g_{0}^{k i},
$$

we see that $(2.2)$ is valid. Similarly, we see that for the metric tensor $g_{\mu}^{i j}=$ $\frac{1}{\operatorname{det}(\mu)} \mu_{k}^{j} g_{0}^{k i}$, we have

$$
\mathcal{B}=*_{0}(\mu H)^{b}=*_{\mu} \mathcal{H}
$$

Hence, the constitutive relations take the form

$$
\mathcal{D}(x, t)=*_{\epsilon} \mathcal{E}(x, t), \quad \mathcal{B}(x, t)=*_{\mu} \mathcal{H}(x, t) .
$$

We consider the waves that satisfy the initial conditions

$$
\left.B(x, t)\right|_{t=-\tau}=0,\left.\quad D(x, t)\right|_{t=-\tau}=0, \quad \tau>0,
$$

Applying the divergence operator to (1.1), we have

$$
\operatorname{div} B(x, t)=0, \operatorname{div} D(x, t)=0, \quad t \in \mathbb{R}, \quad x \in M
$$

In terms of differential forms these equations imply that

$$
d \mathcal{B}=0, \quad d \mathcal{D}=0
$$

In the further considerations, we will use only the pair $(\mathcal{E}, \mathcal{B})$ and denote it by $\left(\omega^{1}, \omega^{2}\right)$, where $\omega^{1}=\mathcal{E}$ and $\omega^{2}=\mathcal{B}$. The compatibility conditions $(2.3)$ 
imply that

$$
d \omega^{2}=0, \quad d *_{\epsilon} \omega^{1}=0 .
$$

It follows from (2.1) that

$$
\omega_{t}^{2}=-d \omega^{1}, \quad \omega_{t}^{1}=*_{\epsilon} d *_{\mu} \omega^{2}
$$

Let us consider the following codifferentials,

$$
\delta_{\epsilon, \mu} \omega^{2}=*_{\epsilon} d *_{\mu} \omega^{2}, \quad \delta_{\mu, \epsilon} \omega^{k}=-*_{\mu} d *_{\epsilon} \omega^{k}, k=1,3 .
$$

Then (2.4) and (2.5) yield

$$
\begin{array}{ll}
\omega_{t}^{1}=\delta_{\epsilon, \mu} \omega^{2}, & \delta_{\mu, \epsilon} \omega^{1}=0, \\
\omega_{t}^{2}=-d \omega^{1}, & d \omega^{2}=0 .
\end{array}
$$

These equations are called Maxwell's equations for forms in the divergence free case on a Riemannian manifold $M$.

We shall now extend the above equations to the full bundle of exterior differential forms $\Lambda T^{*} M$. To this end, we introduce auxiliary forms, $\omega^{0} \in C^{\infty}(M)$ and $\omega^{3} \in C^{\infty}\left(M, \Lambda^{3} T^{*} M\right)$, which vanish in the electromagnetic theory, by

$$
\omega_{t}^{0}=\delta_{\mu, \epsilon} \omega^{1}, \quad \omega_{t}^{3}=-d \omega^{2} .
$$

Since $\omega^{0}=0$ and $\omega^{3}=0$ in the electromagnetic theory, we can modify equations (2.7) to have

$$
\begin{array}{ll}
\omega_{t}^{1}=-d \omega^{0}+\delta_{\epsilon, \mu} \omega^{2}, & \omega_{t}^{3}=-d \omega^{2}, \\
\omega_{t}^{2}=-d \omega^{1}+\delta_{\mu, \epsilon} \omega^{3}, & \omega_{t}^{0}=\delta_{\mu, \epsilon} \omega^{1},
\end{array}
$$

or, in the matrix form,

$$
\omega_{t}+D \omega=0
$$

where $\omega=\left(\omega^{0}, \omega^{1}, \omega^{2}, \omega^{3}\right)$ and the operator $D$ is given by

$$
D=\left(\begin{array}{cccc}
0 & -\delta_{\mu, \epsilon} & 0 & 0 \\
d & 0 & -\delta_{\epsilon, \mu} & 0 \\
0 & d & 0 & -\delta_{\mu, \epsilon} \\
0 & 0 & d & 0
\end{array}\right)
$$


Equations (2.8) and (2.9) are called the complete Maxwell system. Notice that the operator $D$ is of the Dirac type - see also the proof of Proposition 2.1 below.

\subsection{Function spaces}

Define the $L^{2}$-inner product in the space $C^{\infty}\left(M, \Lambda^{k} T^{*} M\right)$ as follows,

$$
\begin{aligned}
& \left(\omega^{k}, \eta^{k}\right)_{L_{\mu}^{2}}=\int_{M} \omega^{k} \wedge *_{\mu} \eta^{k}, \quad k=0,2, \\
& \left(\omega^{k}, \eta^{k}\right)_{L_{\epsilon}^{2}}=\int_{M} \omega^{k} \wedge *_{\epsilon} \eta^{k}, \quad k=1,3,
\end{aligned}
$$

and denote by $L^{2}\left(M, \Lambda^{k} T^{*} M\right)$ the completion of $C^{\infty}\left(M, \Lambda^{k} T^{*} M\right)$ in the corresponding norm. In the complexified case, we take the corresponding sesquilinear extension of the inner product. We denote by $H^{s}\left(M, \Lambda^{k} T^{*} M\right)$ the standard Sobolev space of $k$-forms.

The natural domain of the exterior differential $d$ in $L^{2}\left(M, \Lambda^{k} T^{*} M\right)$ is

$$
H\left(d, \Lambda^{k} T^{*} M\right)=\left\{\omega^{k} \in L^{2}\left(M, \Lambda^{k} T^{*} M\right): d \omega^{k} \in L^{2}\left(M, \Lambda^{k+1} T^{*} M\right)\right\},
$$

and we define

$$
H\left(\delta_{\epsilon, \mu}, \Lambda^{k} T^{*} M\right)=\left\{\omega^{k} \in L^{2}\left(M, \Lambda^{k} T^{*} M\right): \delta_{\epsilon, \mu} \omega^{k} \in L^{2}\left(M, \Lambda^{k-1} T^{*} M\right)\right\},
$$

and similarly for $\delta_{\mu, \epsilon}$.

Let $i^{*}: C^{\infty}\left(M, \Lambda^{k} T^{*} M\right) \rightarrow C^{\infty}\left(\partial M, \Lambda^{k} T^{*} M\right)$ be the pull-back of the imbedding $i: \partial M \rightarrow M$. Then we define the tangential trace of $k$-forms as

$$
\mathbf{t}: C^{\infty}\left(M, \Lambda^{k} T^{*} M\right) \rightarrow C^{\infty}\left(\partial M, \Lambda^{k} T^{*} M\right), \quad \mathbf{t} \omega^{k}=i^{*} \omega^{k}, \quad k=0,1,2,
$$

and the normal trace as

$$
\begin{gathered}
\mathbf{n}: C^{\infty}\left(M, \Lambda^{k} T^{*} M\right) \rightarrow C^{\infty}\left(\partial M, \Lambda^{3-k} T^{*} M\right), \\
\mathbf{n} \omega^{k}=i^{*}\left(*_{\epsilon} \omega^{k}\right), k=1,3, \quad \mathbf{n} \omega^{2}=i^{*}\left(*_{\mu} \omega^{2}\right) .
\end{gathered}
$$

Set

$$
\left\langle\mathbf{t} \omega^{k}, \mathbf{n} \eta^{k+1}\right\rangle=\int_{\partial M} \mathbf{t} \omega^{k} \wedge \mathbf{n} \eta^{k+1}, \quad k=0,1,2 .
$$


With this notation, Stokes' formulae for differential forms can be written as

$$
\begin{aligned}
& \left(d \omega^{0}, \eta^{1}\right)_{L_{\epsilon}^{2}}-\left(\omega^{0}, \delta_{\mu, \epsilon} \eta^{1}\right)_{L_{\mu}^{2}}=\left\langle\mathbf{t} \omega^{0}, \mathbf{n} \eta^{1}\right\rangle \\
& \left(d \omega^{1}, \eta^{2}\right)_{L_{\mu}^{2}}-\left(\omega^{1}, \delta_{\epsilon, \mu} \eta^{2}\right)_{L_{\epsilon}^{2}}=\left\langle\mathbf{t} \omega^{1}, \mathbf{n} \eta^{2}\right\rangle \\
& \left(d \omega^{2}, \eta^{3}\right)_{L_{\epsilon}^{2}}-\left(\omega^{2}, \delta_{\mu, \epsilon} \eta^{3}\right)_{L_{\mu}^{2}}=\left\langle\mathbf{t} \omega^{2}, \mathbf{n} \eta^{3}\right\rangle .
\end{aligned}
$$

Using (2.9) and (2.10), we obtain

$$
(D \omega, \eta)_{L^{2}}+(\omega, D \eta)_{L^{2}}=\langle\mathbf{t} \omega, \mathbf{n} \eta\rangle+\langle\mathbf{t} \eta, \mathbf{n} \omega\rangle
$$

where $\mathbf{t} \omega=\left(\mathbf{t} \omega^{0}, \mathbf{t} \omega^{1}, \mathbf{t} \omega^{2}\right), \mathbf{n} \omega=\left(\mathbf{n} \omega^{1}, \mathbf{n} \omega^{2}, \mathbf{n} \omega^{3}\right)$, and

$$
\langle\mathbf{t} \omega, \mathbf{n} \eta\rangle=\left\langle\mathbf{t} \omega^{0}, \mathbf{n} \eta^{1}\right\rangle+\left\langle\mathbf{t} \omega^{1}, \mathbf{n} \eta^{2}\right\rangle+\left\langle\mathbf{t} \omega^{2}, \mathbf{n} \eta^{3}\right\rangle
$$

Here we take $\omega, \eta \in \mathcal{H}$, where

$$
\begin{aligned}
\mathcal{H}= & H\left(d, \Lambda^{0} T^{*} M\right) \times\left[H\left(d, \Lambda^{1} T^{*} M\right) \cap H\left(\delta_{\mu, \epsilon}, \Lambda^{1} T^{*} M\right)\right] \\
& \times\left[H\left(d, \Lambda^{2} T^{*} M\right) \cap H\left(\delta_{\epsilon, \mu}, \Lambda^{2} T^{*} M\right)\right] \times H\left(\delta_{\mu, \epsilon}, \Lambda^{3} T^{*} M\right) .
\end{aligned}
$$

It will be convenient to write $\delta$ to stand for both $\delta_{\mu, \epsilon}$ and $\delta_{\epsilon, \mu}$, when no risk of ambiguity is possible. There are well defined extensions of the boundary trace operators $\mathbf{t}$ and $\mathbf{n}$ to the spaces $H\left(d, \Lambda^{k} T^{*} M\right)$ and $H\left(\delta, \Lambda^{k} T^{*} M\right)$, see $[25]$.

Lemma 2.1. The operators $\mathbf{t}$ and $\mathbf{n}$ can be extended to continuous surjective maps

$$
\begin{aligned}
\mathbf{t}: H\left(d, \Lambda^{k} T^{*} M\right) & \rightarrow H^{-1 / 2}\left(d, \partial M, \Lambda^{k} T^{*} M\right), \\
\mathbf{n}: H\left(\delta, \Lambda^{k+1} T^{*} M\right) & \rightarrow H^{-1 / 2}\left(d, \partial M, \Lambda^{2-k} T^{*} M\right),
\end{aligned}
$$

where $H^{-1 / 2}\left(d, \partial M, \Lambda^{k} T^{*} M\right)$ is given by

$$
\left\{\omega^{k} \in H^{-1 / 2}\left(\partial M, \Lambda^{k} T^{*} M\right): d \omega^{k} \in H^{-1 / 2}\left(\partial M, \Lambda^{k+1} T^{*} M\right)\right\}
$$

Let $H_{t}\left(d, \Lambda^{k} T^{*} M\right)$ stand for the kernel of $(2.12)$, and $H_{n}\left(\delta, \Lambda^{k+1} T^{*} M\right)$ will denote the kernel of the operator (2.13).

Using (2.10), we can verify the following result in a standard way, see also [16, Lemma 1.3]. 
Lemma 2.2. The Hilbert space adjoint of

$$
d: L^{2}\left(M, \Lambda^{0} T^{*} M\right) \rightarrow L^{2}\left(M, \Lambda^{1} T^{*} M\right)
$$

equipped with the domain $H_{t}\left(d, \Lambda^{0} T^{*} M\right)$, is the operator $\delta_{\mu, \epsilon}$ with the domain $H\left(\delta_{\mu, \epsilon}, \Lambda^{1} T^{*} M\right)$. The Hilbert space adjoint of

$$
\delta_{\mu, \epsilon}: L^{2}\left(M, \Lambda^{1} T^{*} M\right) \rightarrow L^{2}\left(M, \Lambda^{0} T^{*} M\right)
$$

equipped with the domain $H\left(\delta_{\mu, \epsilon}, \Lambda^{1} T^{*} M\right)$, is the operator $d$ with the domain $H_{t}\left(d, \Lambda^{0} T^{*} M\right)$.

It is clear that analogous statements hold for the operators $d$ and $\delta$, acting on forms of higher degree.

We shall need the following result.

Proposition 2.1. $\quad$ (i) The operator D, given by (2.9) and equipped with the domain

$$
\begin{aligned}
\mathcal{D}(D)= & H_{t}\left(d, \Lambda^{0} T^{*} M\right) \times\left[H_{t}\left(d, \Lambda^{1} T^{*} M\right) \cap H\left(\delta_{\mu, \epsilon}, \Lambda^{1} T^{*} M\right)\right] \\
& \times\left[H_{t}\left(d, \Lambda^{2} T^{*} M\right) \cap H\left(\delta_{\epsilon, \mu}, \Lambda^{2} T^{*} M\right)\right] \times H\left(\delta_{\mu, \epsilon}, \Lambda^{3} T^{*} M\right),
\end{aligned}
$$

is skew-adjoint on $L^{2}$.

(ii) The spectrum of the operator $D$ with the domain $\mathcal{D}(D)$ is discrete.

(iii) The operator $D$ is an elliptic differential operator in the interior of $M$.

Proof. (i). Using the definition of the domain of the adjoint and Lemma 2.2 , we obtain that $\mathcal{D}\left(D^{*}\right)=\mathcal{D}(D)$. The skew-adjointness of $D$ then follows from (2.11), which holds for $\omega, \eta \in \mathcal{D}(D)$.

(ii). In view of Gaffney's inequality [26, Corollary 2.1.6],

$$
\begin{aligned}
& H_{t}\left(d, \Lambda^{k} T^{*} M\right) \cap H\left(\delta, \Lambda^{k} T^{*} M\right) \\
& \quad=\left\{\omega^{k} \in H^{1}\left(M, \Lambda^{k} T^{*} M\right): \mathbf{t} \omega^{k}=0\right\}, k=1,2,
\end{aligned}
$$

together with the Sobolev embedding, we conclude that the imbedding $\mathcal{D}(D) \hookrightarrow L^{2}$ is compact. Hence, the spectrum of $\mathcal{D}$ is discrete. 
(iii). It suffices to show the ellipticity of $D^{2}$. Since $\delta_{\mu, \epsilon} \delta_{\epsilon, \mu}=0$ and $\delta_{\epsilon, \mu} \delta_{\mu, \epsilon}=$ 0 , we get

$$
D^{2}=\left(\begin{array}{cccc}
-\delta_{\mu, \epsilon} d & 0 & 0 & 0 \\
0 & -d \delta_{\mu, \epsilon}-\delta_{\epsilon, \mu} d & 0 & 0 \\
0 & 0 & -d \delta_{\epsilon, \mu}-\delta_{\mu, \epsilon} d & 0 \\
0 & 0 & 0 & -d \delta_{\mu, \epsilon}
\end{array}\right)
$$

The operator $D^{2}$ enjoys the following coercive estimate,

$$
\left(D^{2} \omega, \omega\right)_{L^{2}} \geq C_{1}\|\omega\|_{H^{1}}^{2}-C_{2}\|\omega\|_{L^{2}}^{2}, C_{1}>0,
$$

where $\omega=\left(\omega^{0}, \omega^{1}, \omega^{2}, \omega^{3}\right)$ and $\omega^{k} \in C_{0}^{\infty}\left(M, \Lambda^{k} T^{*} M\right), k=0,1,2,3$.

When proving (2.14), notice that, for $\omega^{1} \in C_{0}^{\infty}\left(M, \Lambda^{1} T^{*} M\right)$,

$$
\left(\left(d \delta_{\mu, \epsilon}+\delta_{\epsilon, \mu} d\right) \omega^{1}, \omega^{1}\right)_{L^{2}}=\left\|\delta_{\mu, \epsilon} \omega^{1}\right\|_{L^{2}}^{2}+\left\|d \omega^{1}\right\|_{L^{2}}^{2}
$$

An application of Gaffney's inequality gives that

$$
\left\|\omega^{1}\right\|_{H^{1}} \leq C(M)\left(\left\|\omega^{1}\right\|_{L^{2}}+\left\|d \omega^{1}\right\|_{L^{2}}+\left\|\delta_{\mu, \epsilon} \omega^{1}\right\|_{L^{2}}\right)
$$

where $C(M)>0$ is a constant. The estimate (2.14) follows, since the treatment of forms of degrees different from 1 is analogous. See also [7] for a different proof of coercivity. The ellipticity of $D^{2}$ now follows from the coercivity estimate (2.14), see e.g., [21].

\subsection{Betti numbers and the Euler characteristic of a manifold with boundary}

Let $\left(M, g_{0}\right)$ be an orientable compact Riemannian manifold of dimension 3 with boundary. The space

$$
\mathcal{H}^{k}(M)=\left\{\omega \in L^{2}\left(M, \Lambda^{k} T^{*} M\right): d \omega=0, d * g_{0} \omega=0\right\}
$$

is called the space of harmonic fields. Notice that this space is infinite dimensional for $1 \leq k \leq 2$, see [26, Theorem 3.4.2]. Moreover, it is well-known that harmonic fields are $C^{\infty}$-smooth in the interior of $M$. The following two finite 
dimensional subspaces are distinguished in $\mathcal{H}^{k}(M)$ :

$$
\begin{aligned}
& \mathcal{H}_{D}^{k}(M)=\left\{\omega \in \mathcal{H}^{k}(M): \mathbf{t} \omega=0\right\} \quad \text { and } \\
& \mathcal{H}_{N}^{k}(M)=\left\{\omega \in \mathcal{H}^{k}(M): i^{*}\left(*_{g_{0}} \omega\right)=0\right\}
\end{aligned}
$$

which are called the Dirichlet and Neumann harmonic fields, respectively. It follows from the Hodge theory that the dimensions of the spaces $\mathcal{H}_{D}^{k}(M)$ and $\mathcal{H}_{N}^{k}(M)$ are independent of the choice of the metric $g_{0}$. For our purposes, we shall have to specify the choice of the Hodge star operator in the definition of $\mathcal{H}^{k}(M)$, according to the definition of the codifferential given in (2.6),

$$
\begin{aligned}
& \mathcal{H}^{2}(M)=\left\{\omega \in L^{2}\left(M, \Lambda^{2} T^{*} M\right): d \omega=0, d *_{\mu} \omega=0\right\} \\
& \mathcal{H}^{k}(M)=\left\{\omega \in L^{2}\left(M, \Lambda^{k} T^{*} M\right): d \omega=0, d *_{\epsilon} \omega=0\right\}, k=1,3 .
\end{aligned}
$$

Recall [10] that the space $\mathcal{H}_{N}^{k}(M)$ is isomorphic to the $k$ th homology group of the manifold $H_{k}(M ; \mathbb{R})$ and $\mathcal{H}_{D}^{k}(M)$ is isomorphic to the $k$ th relative homology group $H_{k}(M, \partial M ; \mathbb{R})$. The Poincaré-Lefschetz duality states the existence of the following isomorphism,

$$
H_{k}(M ; \mathbb{R}) \simeq H_{3-k}(M, \partial M ; \mathbb{R}), \quad k=0,1,2,3 .
$$

The $k$ th absolute Betti number of the manifold $M$ is given by

$$
\beta_{k}(M)=\operatorname{dim} \mathcal{H}_{N}^{k}(M), \quad k=0,1,2,3
$$

and the $k$ th relative Betti number of $M$ is defined by

$$
\beta_{k}(M, \partial M)=\operatorname{dim} \mathcal{H}_{D}^{k}(M), \quad k=0,1,2,3
$$

Being one of the simplest topological invariants, the Betti numbers carry a basic amount of information about the topology of a manifold in question. The Betti numbers $\beta_{0}(M)$ and $\beta_{3}(M)$ admit a particularly straightforward geometric interpretation. Namely, $\beta_{0}(M)$ counts the number of the connected components of $M$ and $\beta_{3}(M)$ gives the number of the oriented components of $M$ without boundary. Assuming that the manifold $M$ is connected, we have $\beta_{0}(M)=1$ and $\beta_{3}(M)=0$. As for the first Betti number $\beta_{1}(M)$, it is at least as large as the total number of handles of $\partial M$, see [5, Theorem 5.1.9]. 
The Euler characteristic is defined by

$$
\chi(M)=\beta_{3}(M)-\beta_{2}(M)+\beta_{1}(M)-\beta_{0}(M) .
$$

It is known [8, Corollary 8.8] that the Euler characteristics of a compact three-manifold and its boundary are related by

$$
\chi(\partial M)=2 \chi(M)
$$

Notice finally that if $M$ is a connected compact orientable three-manifold with vanishing Euler characteristic, then either the manifold $M$ is closed or its boundary is a disjoint union of tori.

\subsection{Boundary data for inverse problems}

Let $\Gamma \subset \partial M$ be an open subset of the boundary $\partial M$. Consider the following initial boundary value problem,

$$
\begin{aligned}
\left(\partial_{t}+D\right) \omega(x, t) & =0 \quad \text { in } \quad M \times \mathbb{R} \\
\left.\mathbf{t} \omega\right|_{\partial M \times \mathbb{R}} & =f \in C_{0}^{\infty}\left(\mathbb{R}_{-}, C_{0}^{\infty}\left(\Gamma, \Lambda T^{*} M\right)\right), \\
\left.\omega\right|_{t=-\tau_{f}} & =0
\end{aligned}
$$

where $\tau_{f}>0$ is such that inf supp $(f)>-\tau_{f}$. Following [16], we shall define a solution of (2.16) in the following way. Let $E$ be a right inverse to the trace mapping $\mathbf{t}$ such that $E f\left(-\tau_{f}\right)=0$. We set

$$
\omega^{f}(t)=E f(t)-\int_{-\tau_{f}}^{t} \mathrm{e}^{-(t-s) D}\left(\partial_{s}+D\right) E f(s) d s .
$$

Here $\mathrm{e}^{-t D}$ is the unitary group, generated by the self-adjoint operator $D / i$.

Associated to the problem (2.16) is the response operator,

$$
R_{\Gamma}:\left.f \mapsto \mathbf{n} \omega^{f}\right|_{\Gamma \times \mathbb{R}_{-}}
$$

The first main result of this work is the following theorem.

Theorem 2.1. Assume that we are given an open subset $\Gamma \subset \partial M$ and the response operator $R_{\Gamma}$ for any $f \in C_{0}^{\infty}\left(\mathbb{R}_{-}, C_{0}^{\infty}\left(\Gamma, \Lambda T^{*} M\right)\right)$. These data determine the Betti numbers of the manifold $M$.

The proof of Theorem 2.1 will be given in Section 3 . 
Let us now return to the physical Maxwell's equations

$$
\begin{aligned}
\omega_{t}^{1} & =\delta_{\epsilon, \mu} \omega^{2}, \quad \delta_{\mu, \epsilon} \omega^{1}=0, \\
\omega_{t}^{2} & =-d \omega^{1}, \quad d \omega^{2}=0, \\
\mathbf{t} \omega^{1} & =h \in C_{0}^{\infty}\left(\mathbb{R}_{-}, C_{0}^{\infty}\left(\Gamma, \Lambda^{1} T^{*} M\right)\right), \\
\left.\omega\right|_{t<-\tau_{h}} & =0 .
\end{aligned}
$$

As explained in [16], the solution to (2.18) is obtained from (2.17) by choosing the boundary source $f$ in $(2.16)$ as

$$
f=\left(0, h,-\int_{-\tau_{h}}^{t} d h\left(t^{\prime}\right) d t^{\prime}\right) .
$$

The response operator for (2.18) is defined by

$$
\widetilde{R}_{\Gamma}:\left.h \mapsto \mathbf{n} \omega^{h, 2}\right|_{\Gamma \times \mathbb{R}_{-}},
$$

where $\omega^{h}$ is the solution to (2.18). Notice that in the classical terminology of electric and magnetic fields, the response operator $\widetilde{R}_{\Gamma}$ maps the tangential component of the electric field $n \times\left. E\right|_{\Gamma \times \mathbb{R}_{-}}$to the tangential component of the magnetic field $n \times\left. H\right|_{\Gamma \times \mathbb{R}_{-}}$.

Theorem 2.2. Given an open subset $\Gamma \subset \partial M$ and the response operator $\widetilde{R}_{\Gamma}$ for any $h \in C_{0}^{\infty}\left(\mathbb{R}_{-}, C_{0}^{\infty}\left(\Gamma, \Lambda^{1} T^{*} M\right)\right)$, the first absolute Betti number $\beta_{1}(M)$ of the manifold $M$ can be determined.

Corollary 2.1. The knowledge of the boundary $\partial M$ and the response operator $\widetilde{R}_{\Gamma}, \Gamma \subset \partial M$, for any $h \in C_{0}^{\infty}\left(\mathbb{R}_{-}, C_{0}^{\infty}\left(\Gamma, \Lambda^{1} T^{*} M\right)\right)$, determines the first and the second absolute Betti numbers $\beta_{1}(M)$ and $\beta_{2}(M)$ of $M$.

Corollary 2.1 follows from Theorem 2.2 together with (2.15).

\subsection{Examples}

The following two examples illustrate the significance of our results for the determination of the topological structure of an unknown object from the boundary measurements. This may have applications to practical situations, where the structure of complicated voids in an unknown object is to be recovered.

Example 2.1. Let $M \subset \mathbb{R}^{3}$ be obtained from a large ball by removing a finite number of pairwise disjoint solid tori. Then the first absolute Betti 
number of $M$ is equal to the number of the removed solid tori. Thus, measuring the response operator on a portion of the boundary sphere, we can recover the total number of the removed tori.

Example 2.2. Consider a solid torus $\mathrm{ST}=S^{1} \times D^{2} \subset \mathbb{R}^{3}$, where $S^{1}$ is a unit circle and $D^{2}$ is a closed two-dimensional disc. The boundary of ST is a two-dimensional torus and since $D^{2}$ is contractible, it follows that the first absolute Betti number of ST is equal to 1 . Let $M$ be the connected sum of $k$ copies of solid tori ST. Here we may recall that a connected sum of two manifolds, possibly with boundary, is a manifold formed by deleting a ball in the interior of each of the manifolds and gluing together the resulting boundary spheres. The boundary of $M$ is a disjoint union of $k$ copies of two-dimensional tori. It is known that for manifolds of dimension three and higher, the first absolute Betti number of the connected sum is the sum of the first absolute Betti numbers of the summands. Therefore, the first absolute Betti number of $M$ is equal to $k$. It follows from our results that performing measurements on a portion of the boundary of the manifold $M$, we are able to recover the total number of the solid tori.

\section{Proof of Theorem 2.1}

\subsection{Inner products}

Let $\omega^{f}(t)=\omega^{f}(x, t)$ be the solution to (2.16). We shall need the following Blagovestchenskii type result, see [3] for such results for one-dimensional inverse problems.

Theorem 3.1. For any $f, h \in C_{0}^{\infty}\left(\mathbb{R}_{-}, C_{0}^{\infty}\left(\Gamma, \Lambda T^{*} M\right)\right)$, the knowledge of $\Gamma \subset \partial M$ and the response operator $R_{\Gamma}$ allows us to evaluate the inner products

$$
\left(\omega^{f, k}(t), \omega^{h, k}(s)\right)_{L^{2}}, \quad k=0,1,2,3, \quad \text { for } \quad s, t \geq 0 .
$$

Proof. From (2.17), we obtain that

$$
\omega^{f}(t)=\omega^{f_{t}}(-1), \quad t \geq 0,
$$

where $f_{t}=f(\cdot+t+1), f_{t} \in C_{0}^{\infty}\left(\mathbb{R}_{-}, C_{0}^{\infty}\left(\Gamma, \Lambda T^{*} M\right)\right)$. Therefore, the knowledge of the operator $R_{\Gamma}$ is equivalent to the knowledge of the operator 


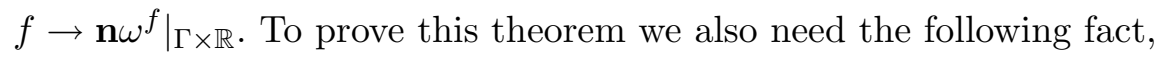

$$
\mathbf{t}\left(d \omega^{k}\right)=d\left(\mathbf{t} \omega^{k}\right), \quad k=0,1,2,3
$$

see [26, Proposition 1.2.6]. This implies that

$$
\begin{aligned}
& \mathbf{n} \delta_{\epsilon, \mu} \omega^{2}=\mathbf{t}\left(*_{\epsilon} *_{\epsilon} d *_{\mu} \omega^{2}\right)=d \mathbf{t}\left(*_{\mu} \omega^{2}\right)=d \mathbf{n} \omega^{2} \\
& \mathbf{n} \delta_{\mu, \epsilon} \omega^{3}=\mathbf{t}\left(*_{\mu}(-1) *_{\mu} d *_{\epsilon} \omega^{3}\right)=-d \mathbf{t}\left(*_{\epsilon} \omega^{3}\right)=-d \mathbf{n} \omega^{3} .
\end{aligned}
$$

Set $I^{k}(s, t)=\left(\omega^{f, k}(t), \omega^{h, k}(s)\right)_{L^{2}}, \quad k=0, \ldots, 3$. Then using Stokes' formulae, we get

$$
\begin{aligned}
\left(\partial_{s}^{2}-\partial_{t}^{2}\right) I^{0}(s, t)= & \left(\omega^{f, 0}(t), \partial_{s}^{2} \omega^{h, 0}(s)\right)-\left(\partial_{t}^{2} \omega^{f, 0}(t), \omega^{h, 0}(s)\right) \\
= & -\left(\omega^{f, 0}(t), \delta_{\mu, \epsilon} d \omega^{h, 0}(s)\right)+\left(\delta_{\mu, \epsilon} d \omega^{f, 0}(t), \omega^{h, 0}(s)\right) \\
= & \left\langle\mathbf{t} \omega^{f, 0}(t), \mathbf{n} d \omega^{h, 0}(s)\right\rangle-\left\langle\mathbf{t} \omega^{h, 0}(s), \mathbf{n} d \omega^{f, 0}(t)\right\rangle \\
= & -\left\langle\mathbf{t} \omega^{f, 0}(t), \partial_{s} \mathbf{n} \omega^{h, 1}(s)\right\rangle+\left\langle\mathbf{t} \omega^{f, 0}(t), \mathbf{n} \delta_{\epsilon, \mu} \omega^{h, 2}(s)\right\rangle \\
& +\left\langle\mathbf{t} \omega^{h, 0}(s), \partial_{t} \mathbf{n} \omega^{f, 1}(t)\right\rangle-\left\langle\mathbf{t} \omega^{h, 0}(s), \mathbf{n} \delta_{\epsilon, \mu} \omega^{f, 2}(t)\right\rangle \\
= & -\left\langle\mathbf{t} \omega^{f, 0}(t), \partial_{s} \mathbf{n} \omega^{h, 1}(s)\right\rangle+\left\langle\mathbf{t} \omega^{f, 0}(t), d \mathbf{n} \omega^{h, 2}(s)\right\rangle \\
& +\left\langle\mathbf{t} \omega^{h, 0}(s), \partial_{t} \mathbf{n} \omega^{f, 1}(t)\right\rangle-\left\langle\mathbf{t} \omega^{h, 0}(s), d \mathbf{n} \omega^{f, 2}(t)\right\rangle .
\end{aligned}
$$

Similarly,

$$
\begin{aligned}
\left(\partial_{s}^{2}-\right. & \left.\partial_{t}^{2}\right) I^{1}(s, t) \\
= & \left(\omega^{f, 1}(t), \partial_{s}^{2} \omega^{h, 1}(s)\right)-\left(\partial_{t}^{2} \omega^{f, 1}(t), \omega^{h, 1}(s)\right) \\
= & -\left\langle\partial_{s} \mathbf{t} \omega^{h, 0}(s), \mathbf{n} \omega^{f, 1}(t)\right\rangle-\left\langle\mathbf{t} \omega^{f, 1}(t), \partial_{s} \mathbf{n} \omega^{h, 2}(s)+d \mathbf{n} \omega^{h, 3}(s)\right\rangle \\
& +\left\langle\partial_{t} \mathbf{t} \omega^{f, 0}(t), \mathbf{n} \omega^{h, 1}(s)\right\rangle+\left\langle\mathbf{t} \omega^{h, 1}(s), \partial_{t} \mathbf{n} \omega^{f, 2}(t)+d \mathbf{n} \omega^{f, 3}(t)\right\rangle \\
\left(\partial_{s}^{2}-\right. & \left.\partial_{t}^{2}\right) I^{2}(s, t) \\
= & \left(\omega^{f, 2}(t), \partial_{s}^{2} \omega^{h, 2}(s)\right)-\left(\partial_{t}^{2} \omega^{f, 2}(t), \omega^{h, 2}(s)\right) \\
= & -\left\langle\partial_{s} \mathbf{t} \omega^{h, 1}(s)+d \mathbf{t} \omega^{h, 0}(s), \mathbf{n} \omega^{f, 2}(t)\right\rangle-\left\langle\mathbf{t} \omega^{f, 2}(t), \partial_{s} \mathbf{n} \omega^{h, 3}(s)\right\rangle \\
& +\left\langle\partial_{t} \mathbf{t} \omega^{f, 1}(t)+d \mathbf{t} \omega^{f, 0}(t), \mathbf{n} \omega^{h, 2}(s)\right\rangle+\left\langle\mathbf{t} \omega^{h, 2}(s), \partial_{t} \mathbf{n} \omega^{f, 3}(t)\right\rangle \\
\left(\partial_{s}^{2}-\right. & \left.\partial_{t}^{2}\right) I^{3}(s, t) \\
= & \left(\omega^{f, 3}(t), \partial_{s}^{2} \omega^{h, 3}(s)\right)-\left(\partial_{t}^{2} \omega^{f, 3}(t), \omega^{h, 3}(s)\right) \\
= & -\left\langle\partial_{s} \mathbf{t} \omega^{h, 2}(s)+d \mathbf{t} \omega^{h, 1}(s), \mathbf{n} \omega^{f, 3}(t)\right\rangle \\
& +\left\langle\partial_{t} \mathbf{t} \omega^{f, 2}(t)+d \mathbf{t} \omega^{f, 1}(t), \mathbf{n} \omega^{h, 3}(s)\right\rangle .
\end{aligned}
$$

Hence $I^{k}(s, t), \quad k=0,1,2,3$, satisfies an inhomogeneous one-dimensional wave equation in the unbounded region $\left\{(s, t) \in \mathbb{R}^{2}: s \geq-\tau_{h}, t \geq-\tau_{f}\right\}$, 
whose right hand side is determined from the knowledge of $\Gamma$ and $R_{\Gamma}$. Since

$$
I^{k}\left(-\tau_{h}, t\right)=I^{k}\left(s,-\tau_{f}\right)=0, \quad \partial_{s} I^{k}\left(-\tau_{h}, t\right)=\partial_{t} I^{k}\left(s,-\tau_{f}\right)=0,
$$

we can determine $I^{k}(s, t)$ in the entire region $s \geq-\tau_{f}, t \geq-\tau_{f}$. The result follows.

\subsection{Controllability result}

In the isotropic setting and the case when $\epsilon(x)=\alpha(x) \mu(x), \alpha(x)>0$, one can use a generalization of Tataru's unique continuation theorem $[9,16]$ to obtain controllability results with sources supported in a finite time interval. As already mentioned in the introduction, such unique continuation results do not seem to be available in the general anisotropic setting. Nevertheless, we shall next show that partial controllability results in the general anisotropic setting on an infinite time interval can be obtained using a unique continuation principle for elliptic systems. As shown below, this turns out to be sufficient for the reconstruction of the Betti numbers.

Let $\mathcal{H}_{D}(M):=\oplus_{k=0}^{3} \mathcal{H}_{D}^{k}(M)$ be the space of all Dirichlet harmonic fields, and let $\Pi: L^{2}\left(M, \Lambda T^{*} M\right) \rightarrow \mathcal{H}_{D}(M)$ be the orthogonal projection.

Theorem 3.2. We have

$$
\left\{\Pi\left(\omega^{f}(0)\right): f \in C_{0}^{\infty}\left(\mathbb{R}_{-}, C_{0}^{\infty}\left(\Gamma, \Lambda T^{*} M\right)\right)\right\}=\mathcal{H}_{D}(M) .
$$

Proof. Let $\eta \in \mathcal{H}_{D}(M)$. If we prove that the orthogonality condition

$$
\left(\omega^{f}(0), \eta\right)_{L^{2}}=0 \quad \text { for all } f \in C_{0}^{\infty}\left(\mathbb{R}_{-}, C_{0}^{\infty}\left(\Gamma, \Lambda T^{*} M\right)\right)
$$

implies that $\eta=0$, then the space $\left\{\Pi\left(\omega^{f}(0)\right): f \in C_{0}^{\infty}\left(\mathbb{R}_{-}, C_{0}^{\infty}\left(\Gamma, \Lambda T^{*} M\right)\right)\right\}$ is dense in $\mathcal{H}_{D}(M)$. Since the latter space is finite dimensional, the claim follows.

As $D \eta=0$, we shall view $\eta(x)$ as the solution to the following problem, dual to $(2.16)$,

$$
\begin{aligned}
\left(-\partial_{t}-D\right) u & =0, \quad \text { in } \quad M \times \mathbb{R}, \\
\left.\mathbf{t} u\right|_{\partial M \times \mathbb{R}} & =0, \\
\left.u\right|_{t=0} & =\eta .
\end{aligned}
$$

Using (2.11), we have

$$
\partial_{t}\left(\omega^{f}, u\right)_{L^{2}}=-\left(D \omega^{f}, u\right)_{L^{2}}-\left(\omega^{f}, D u\right)_{L^{2}}=-\langle f, \mathbf{n} u\rangle .
$$


Thus,

$$
\int_{-\tau_{f}}^{0}\langle f, \mathbf{n} u\rangle d t=-\left(\omega^{f}(0), \eta\right)_{L^{2}}+\left(\omega^{f}\left(-\tau_{f}\right), u\left(-\tau_{f}\right)\right)_{L^{2}}=0 .
$$

The choice of $-\tau_{f}$ implies that

$$
\int_{\mathbb{R}_{-}}\langle f, \mathbf{n} u\rangle d t=0
$$

for all $f \in C_{0}^{\infty}\left(\mathbb{R}_{-}, C_{0}^{\infty}\left(\Gamma, \Lambda T^{*} M\right)\right)$. Thus, $\mathbf{n} u=0$ on $\Gamma \times \mathbb{R}_{-}$.

Now if $\Gamma$ coincides with the whole boundary of the manifold $M$, then we are done, since $\mathcal{H}_{D}^{k}(M) \cap \mathcal{H}_{N}^{k}(M)=\{0\}$, see [26, p. 130].

In the case when $\Gamma$ is a proper open subset of $\partial M$, we proceed as follows. Write $\eta=\left(\eta^{0}, \eta^{1}, \eta^{2}, \eta^{3}\right)$, where $\eta^{k} \in \mathcal{H}_{D}^{k}$. We already know that $\eta^{0}=0$, since $d \eta^{0}=0$ and $\mathbf{t} \eta^{0}=0$. As for $\eta^{1}$, we write

$$
\left(d\left(*_{\epsilon} d *_{\epsilon}\right)+\left(*_{\epsilon} d *_{\epsilon}\right) d\right) \eta^{1}=0
$$

and notice that the Cauchy data of $\eta^{1}$ on $\Gamma$,

$$
\left(\mathbf{t} \eta^{1}, \mathbf{t}\left(*_{\epsilon} \eta^{1}\right), \mathbf{t}\left(*_{\epsilon} d *_{\epsilon} \eta^{1}\right), \mathbf{t}\left(*_{\epsilon} d \eta^{1}\right)\right)
$$

vanishes. Thus, by the unique continuation principle for second-order elliptic systems with diagonal principal part, see [13, Theorem 4.3], we get $\eta^{1}=0$ in $M$. Similar arguments allow us to conclude that $\eta^{2}=\eta^{3}=0$. The proof is complete.

Corollary 3.1. Let $\Pi^{k}: L^{2}\left(M, \Lambda^{k} T^{*} M\right) \rightarrow \mathcal{H}_{D}^{k}(M)$ be the orthogonal projection onto the space of the Dirichlet harmonic $k$-fields. Then

$$
\left\{\Pi^{k}\left(\omega^{f, k}(0)\right): f \in C_{0}^{\infty}\left(\mathbb{R}_{-}, C_{0}^{\infty}\left(\Gamma, \Lambda T^{*} M\right)\right)\right\}=\mathcal{H}_{D}^{k}(M), \quad k=0,1,2,3 .
$$

\subsection{Determination of the Betti numbers of the manifold}

Lemma 3.1. Let $f, h \in C_{0}^{\infty}\left(\mathbb{R}_{-}, C_{0}^{\infty}\left(\Gamma, \Lambda T^{*} M\right)\right)$. Then given the response operator $R_{\Gamma}$, it is possible to find the inner products

$$
\left(\Pi^{k} \omega^{f, k}(0), \omega^{h, k}(0)\right)_{L^{2}}, \quad k=0,1,2,3 .
$$

Proof. Using (2.10), we check by a direct computation that ker $D=\mathcal{H}_{D}(M)$. We can therefore view $\Pi$ as the spectral projection onto the zero eigenspace 
of $D$. Consider the unitary group $\mathrm{e}^{-t D}, t \in \mathbb{R}$, on $L^{2}$. We shall make use of the following essentially well-known formula,

$$
\Pi=\lim _{T \rightarrow+\infty} \frac{1}{T} \int_{0}^{T} \mathrm{e}^{-t D} d t
$$

valid in the sense of strong convergence of operators. When checking (3.3), let

$$
\Pi_{T}=\frac{1}{T} \int_{0}^{T} \mathrm{e}^{-t D} d t \in \mathcal{L}\left(L^{2}, L^{2}\right) .
$$

Since $\left\|\Pi_{T}\right\|_{\mathcal{L}\left(L^{2}, L^{2}\right)} \leq 1$, it suffices to check that $\Pi_{T} x \rightarrow \Pi x$ when $x$ varies in a dense subset of $L^{2}$. We can take this subset to be the set of all finite linear combinations of the eigenfunctions of $D$. To obtain (3.3), we only need to observe that when $\lambda \in \mathbb{R}$,

$$
\lim _{T \rightarrow+\infty} \frac{1}{T} \int_{0}^{T} \mathrm{e}^{i t \lambda} d t= \begin{cases}1 & \text { if } \lambda=0 \\ 0 & \text { if } \lambda \neq 0\end{cases}
$$

Now notice that since supp $(f) \subset \mathbb{R}_{-}$, we have

$$
\mathrm{e}^{-t D} \omega^{f}(0)=\omega^{f}(t), \quad t \geq 0
$$

and therefore,

$$
\Pi \omega^{f}(0)=\lim _{T \rightarrow+\infty} \frac{1}{T} \int_{0}^{T} \omega^{f}(t) d t .
$$

We obtain

$$
\begin{aligned}
\left(\Pi^{k} \omega^{f, k}(0), \omega^{h, k}(0)\right)_{L^{2}} & =\lim _{T \rightarrow+\infty} \frac{1}{T} \int_{0}^{T}\left(\omega^{f, k}(t), \omega^{h, k}(0)\right)_{L^{2}} \\
& =\lim _{T \rightarrow+\infty} \frac{1}{T} \int_{0}^{T}\left(\omega^{f_{t}, k}(0), \omega^{h, k}(0)\right)_{L^{2}}
\end{aligned}
$$

where $f_{t}=f(\cdot+t), f_{t} \in C_{0}^{\infty}\left(\mathbb{R}_{-}, C_{0}^{\infty}\left(\Gamma, \Lambda T^{*} M\right)\right)$. Here we have used that $\omega^{f}(t)=\omega^{f_{t}}(0)$, as follows from (2.17). An application of Theorem 3.1 concludes the proof.

We have the following result which implies Theorem 2.1.

Lemma 3.2. Given $\Gamma \subset \partial M$ and the response operator $R_{\Gamma}$, it is possible to construct a finite number of boundary sources $f_{j} \in C_{0}^{\infty}\left(\mathbb{R}_{-}, C_{0}^{\infty}\left(\Gamma, \Lambda T^{*} M\right)\right)$ such that $\Pi^{k}\left(\omega^{f_{j}, k}(0)\right)$ form a basis of $\mathcal{H}_{D}^{k}(M), 0 \leq k \leq 3$. 
Proof. Let $\left\{h_{j}\right\}_{j=1}^{\infty}$ be a dense countable set in $C_{0}^{\infty}\left(\mathbb{R}_{-}, C_{0}^{\infty}\left(\Gamma, \Lambda T^{*} M\right)\right)$. We can use the Gram-Schmidt orthogonalization procedure to construct the sources $f_{j}$. More precisely, we define $f_{j}$ recursively by

$$
\begin{aligned}
f_{1} & =\frac{h_{1}}{\left(\Pi^{k} \omega^{h_{1}, k}(0), \omega^{h_{1}, k}(0)\right)_{L^{2}}^{1 / 2}}, \\
g_{j} & =h_{j}-\sum_{i=1}^{j-1}\left(\Pi^{k} \omega^{h_{j}, k}(0), \omega^{h_{i}, k}(0)\right)_{L^{2}} f_{i}, \quad j=2,3, \ldots, \\
f_{j} & =\frac{g_{j}}{\left(\Pi^{k} \omega^{g_{j}, k}(0), \omega^{g_{j}, k}(0)\right)_{L^{2}}^{1 / 2}} .
\end{aligned}
$$

When $g_{j}=0$, we remove the corresponding $h_{j}$ from the original sequence and continue the procedure. The number of sources $f_{j}$ produced by the Gram-Schmidt orthogonalization procedure will then be the dimension of $\mathcal{H}_{D}^{k}(M)$, according to Corollary 3.1 .

\section{Proof of Theorem 2.2}

First notice that as in Theorem 3.1, for any $f, h \in C_{0}^{\infty}\left(\mathbb{R}_{-}, C_{0}^{\infty}\left(\Gamma, \Lambda^{1} T^{*} M\right)\right)$, the knowledge of the response operator $\widetilde{R}_{\Gamma}$ allows us to evaluate the inner products,

$$
\left(\omega^{f, k}(t), \omega^{h, k}(s)\right)_{L^{2}}, \quad k=1,2, \quad t, s \geq 0
$$

where $\omega^{f}, \omega^{h}$ are solutions of physical Maxwell's equations (2.18).

We have the following controllability result.

Lemma 4.1. Let $\omega^{f}$ be a solution to physical Maxwell's equations (2.18). Then

$$
\left\{\Pi^{2}\left(\omega^{f, 2}(0)\right): f \in C_{0}^{\infty}\left(\mathbb{R}_{-}, C_{0}^{\infty}\left(\Gamma, \Lambda^{1} T^{*} M\right)\right)\right\}=\mathcal{H}_{D}^{2}(M),
$$

where $\Pi^{2}$ is the orthogonal projection onto the space of the Dirichlet harmonic two-fields.

Proof. Let $\eta^{2} \in \mathcal{H}_{D}^{2}(M)$. Assume that

$$
\left(\omega^{f, 2}(0), \eta^{2}\right)_{L^{2}}=0 \quad \text { for all } f \in C_{0}^{\infty}\left(\mathbb{R}_{-}, C_{0}^{\infty}\left(\Gamma, \Lambda^{1} T^{*} M\right)\right)
$$


Now (2.18) and Stokes' formula imply that

$$
\begin{aligned}
\partial_{t}\left(\omega^{f, 2}(t), \eta^{2}\right)_{L^{2}} & =\left(-d \omega^{f, 1}(t), \eta^{2}\right)_{L^{2}}=-\left(\omega^{1}(t), \delta_{\epsilon, \mu} \eta^{2}\right)_{L^{2}}-\left\langle\mathbf{t} \omega^{f, 1}(t), \mathbf{n} \eta^{2}\right\rangle \\
& =-\left\langle f(t), \mathbf{n} \eta^{2}\right\rangle .
\end{aligned}
$$

Thus,

$$
\int_{\mathbb{R}_{-}}\left\langle f(t), \mathbf{n} \eta^{2}\right\rangle=-\left(\omega^{f, 2}(0), \eta^{2}\right)_{L^{2}}+\left(\omega^{f, 2}\left(-\tau_{f}\right), \eta^{2}\right)_{L^{2}}=0
$$

for all $f \in C_{0}^{\infty}\left(\mathbb{R}_{-}, C_{0}^{\infty}\left(\Gamma, \Lambda^{1} T^{*} M\right)\right)$. Hence, $\mathbf{n} \eta^{2}=0$ on $\Gamma$. Moreover, $\Delta \eta^{2}=0$ on $M$ and $\mathbf{t} \eta^{2}=0$ on $\partial M$. By the unique continuation principle, we get $\eta^{2}=0$.

Proceeding further as in Subsection 3.3, we can recover the first absolute Betti number $\beta_{1}(M)$ from the knowledge of $\Gamma$ and $\widetilde{R}_{\Gamma}$. This completes the proof of Theorem 2.2.

\section{Acknowledgments}

We would to thank Semen Podkorytov for a helpful discussion and providing useful references on topology of manifolds. The research of K.K. was financially supported by the Academy of Finland (project 125599). The research of Y.K. is partially supported by EPSRC Grant EP/F034016/1. The research of M.L. was financially supported by the Academy of Finland Center of Excellence programme 213476.

\section{References}

[1] M. Belishev, V. Isakov, L. Pestov and V. Sharafutdinov, On the reconstruction of a metric from external electromagnetic measurements (Russian) Dokl. Akad. Nauk 372(3) (2000), 298-300.

[2] M. Belishev and V. Sharafutdinov, Dirichlet to Neumann operator on differential forms, Bull. Sci. Math. 132 (2008), 128-145.

[3] A. S. Blagovestchenskii, A one-dimensional inverse boundary value problem for a second order hyperbolic equation, Zap. Naučn. Sem. Leningrad. Otdel. Mat. Inst. Steklov. (LOMI) 15 (1969), 85-90.

[4] P. Caro, P. Ola and M. Salo, Inverse boundary value problem for Maxwell equations with local data, Comm. PDE 34(11) (2009), $1425-1464$. 
[5] D. J. Collins, R. I. Grigorchuk, P. F. Kurchanov and H. Zieschang, Combinatorial group theory and applications to geometry. Translated from the 1990 Russian original by P. M. Cohn, Reprint of the original English edition from the series Encyclopaedia of Mathematical Sciences [Algebra. VII, Encyclopaedia Math. Sci., 58, Springer, Berlin, 1993]. Springer-Verlag, Berlin, 1998, 240.

[6] D. Colton and L. Päivärinta, The uniqueness of a solution to an inverse scattering problem for electromagnetic waves, Arch. Rational Mech. Anal. 119(1) (1992), 59-70.

[7] M. Costabel, A coercive bilinear form for Maxwell's equations, J. Math. Anal. Appl. 157(2) (1991), 527-541.

[8] A. Dold, Lectures on algebraic topology. 2nd ed. Grundlehren der Mathematischen Wissenschaften [Fundamental Principles of Mathematical Sciences], 200, Springer-Verlag, Berlin-New York, 1980, 377.

[9] M. Eller, V. Isakov, G. Nakamura and D. Tataru, Uniqueness and stability in the Cauchy problem for Maxwell and elasticity systems. Nonlinear partial differential equations and their applications, Stud. Math. Appl., 31, North-Holland, Amsterdam, 2002, 329-349.

[10] T. Frankel, The geometry of physics. An introduction. Cambridge University Press, Cambridge, 1997, 654.

[11] A. Greenleaf, Y. Kurylev, M. Lassas and G. Uhlmann, Invisibility and inverse problems, Bull. Amer. Math. Soc. (N.S.) 46(1) (2009), 55-97.

[12] A. Greenleaf, Y. Kurylev, M. Lassas and G. Uhlmann, Full-wave invisibility of active devices at all frequencies, Comm. Math. Phys. 275(3) (2007), 749-789.

[13] V. Isakov, Carleman type estimates and their applications, New analytic and geometric methods in inverse problems, Springer, Berlin, 2004, $93-125$.

[14] C. Kenig, M. Salo and G. Uhlmann, Inverse problems for the anisotropic Maxwell equations, Duke Math. J. 157(2) (2011), 369-419.

[15] Y. Kurylev and M. Lassas, Inverse problems and index formulae for Dirac operators, Adv. Math. 221 (2009), 170-216. 
[16] Y. Kurylev, M. Lassas and E. Somersalo, Maxwell's equations with a polarization independent wave velocity: direct and inverse problems, J. Math. Pures Appl. (9) 86(3) (2006), 237-270.

[17] M. Lassas and G. Uhlmann, On determining a Riemannian manifold from the Dirichlet-to-Neumann map. Ann. Sci. École Norm. Sup. (4) 34(5) (2001), 771-787.

[18] M. Lassas, M. Taylor and G. Uhlmann, The Dirichlet-to-Neumann map for complete Riemannian manifolds with boundary, Comm. Anal. Geom. 11(2) (2003), 207-221.

[19] J. Lee and G. Uhlmann, Determining anisotropic real-analytic conductivities by boundary measurements, Comm. Pure Appl. Math. 42(8) (1989), 1097-1112.

[20] S. McDowall, Boundary determination of material parameters from electromagnetic boundary information, Inverse Problems 13(1) (1997), 153-163

[21] A. Melin, Lower bounds for pseudo-differential operators, Ark. Mat. 9 (1971), 117-140.

[22] P. Ola, L. Päivärinta and E. Somersalo, An inverse boundary value problem in electrodynamics, Duke Math. J. 70 (1993), 617-653.

[23] P. Ola, L. Päivärinta and E. Somersalo, Inverse problems for time harmonic electrodynamics, Inside out: inverse problems, MSRI Publications 47 (2003), 169-191.

[24] P. Ola and E. Somersalo, Electromagnetic inverse problems and generalized Sommerfeld potentials, SIAM J. Appl. Math. 56 (1996), 1129-1145.

[25] L. Paquet, Problèmes mixtes pour le système de Maxwell (French) [Mixed problems for the Maxwell system], Ann. Fac. Sci. Toulouse Math. (5) 4(2) (1982), 103-141.

[26] G. Schwarz, Hodge decomposition - a method for solving boundary value problems, Lecture Notes in Math. 1607, Springer-Verlag, Berlin, 1995.

[27] W. Thirring, A course in mathematical physics, Classical field theory. Translated from the German by Evans M. Harrell. (2), Springer-Verlag, New York-Vienna, 1979, 249. 
Department of Mathematics and Statistics

University of Helsinki, P.O. Box 68,

FI-00014 Helsinki, Finland

E-mail address: katya.krupchyk@helsinki.fi

Department of Mathematics

University College LONDON

Gower Street, London

WC1E 6BT

$\mathrm{UK}$

E-mail address: Y.Kurylev@ucl.ac.uk

Department of Mathematics and Statistics

University of Helsinki, P.O. Box 68,

FI-00014 Helsinki, Finland

E-mail address: matti.lassas@helsinki.fi

ReCEIVED June 26, 2010 
